\title{
The Strong Consistency of the Estimator of Fixed-Design Regression Model under Negatively Dependent Sequences
}

\author{
Xuejun Wang, Meimei Ge, Shuhe Hu, and Xize Wang \\ School of Mathematical Science, Anhui University, Hefei 230601, China \\ Correspondence should be addressed to Xuejun Wang; 07019@ahu.edu.cn
}

Received 25 June 2013; Accepted 7 October 2013

Academic Editor: Michiel Bertsch

Copyright ( 2013 Xuejun Wang et al. This is an open access article distributed under the Creative Commons Attribution License, which permits unrestricted use, distribution, and reproduction in any medium, provided the original work is properly cited.

\begin{abstract}
We study the strong consistency of estimator of fixed design regression model under negatively dependent sequences by using the classical Rosenthal-type inequality and the truncated method. As an application, the strong consistency for the nearest neighbor estimator is obtained.
\end{abstract}

\section{Introduction}

Let $\left\{X_{n}, n \geq 1\right\}$ be a sequence of random variables defined on a fixed probability space $(\Omega, \mathscr{F}, P)$. It is well known that the Rosenthal-type inequality for the partial sum $\sum_{i=1}^{n} X_{i}$ plays an important role in probability limit theory and mathematical statistics. The main purpose of the paper is to investigate the strong consistency of the estimator of fixed design regression model under negatively dependent sequences, by using the Rosenthal-type inequality.

Consider the following fixed design regression model:

$$
Y_{n i}=g\left(x_{n i}\right)+\varepsilon_{n i}, \quad i=1,2, \ldots, n, n \geq 1,
$$

where $x_{n i}$ are known fixed design points from $A$, where $A \subset$ $\mathbb{R}^{p}$ is a given compact set for some $p \geq 1, g(\cdot)$ is an unknown regression function defined on $A$, and $\varepsilon_{n i}$ are random errors. Assume that, for each $n \geq 1,\left(\varepsilon_{n 1}, \varepsilon_{n 2}, \ldots, \varepsilon_{n n}\right)$ have the same distribution as $\left(\varepsilon_{1}, \varepsilon_{2}, \ldots, \varepsilon_{n}\right)$. As an estimator of $g(\cdot)$, the following weighted regression estimator will be considered:

$$
g_{n}(x)=\sum_{i=1}^{n} W_{n i}(x) Y_{n i}, \quad x \in A \subset \mathbb{R}^{p},
$$

where $W_{n i}(x)=W_{n i}\left(x ; x_{n 1}, x_{n 2}, \ldots, x_{n n}\right), i=1,2, \ldots, n$, are the weight function.

The above estimator was first proposed by Georgiev [1] and subsequently has been studied by many authors. For instance, when $\varepsilon_{n i}$ are assumed to be independent, consistency and asymptotic normality have been studied by Georgiev and Greblicki [2], Georgiev [3], and Müller [4] among others. Results for the case when $\varepsilon_{n i}$ are dependent have also been studied by various authors in recent years. Fan [5] extended the work of Georgiev [3] and Müller [4] in the estimation of the regression model to the case which form an $L_{q}$-mixingale sequence for some $1 \leq q \leq 2$. Roussas [6] discussed strong consistency and quadratic mean consistency for $g_{n}(x)$ under mixing conditions. Roussas et al. [7] established asymptotic normality of $g_{n}(x)$ assuming that the errors are from a strictly stationary stochastic process and satisfying the strong mixing condition. Tran et al. [8] discussed again asymptotic normality of $g_{n}(x)$ assuming that the errors form a linear time series, more precisely, a weakly stationary linear process based on a martingale difference sequence. $\mathrm{Hu}$ et al. [9] studied the asymptotic normality for double array sum of linear time series. $\mathrm{Hu}$ et al. [10] gave the mean consistency, complete consistency, and asymptotic normality of regression models with linear process errors. Liang and Jing [11] presented some asymptotic properties for estimates of nonparametric regression models based on negatively associated sequences; Yang et al. [12] generalized the results of Liang and Jing [11] for negatively associated sequences to the case of negatively orthant dependent sequences. Shen [13] presented the Bernstein-type inequality for widely dependent random variables and gave its application to nonparametric regression models, and so forth. The main purpose of this section is to investigate the strong consistency of estimator 
of the fixed design regression model based on negatively dependent random variables.

The concept of negatively dependent random variables was introduced by Lehmann [14] as follows.

A finite collection of random variables $X_{1}, X_{2}, \ldots, X_{n}$ is said to be negatively dependent (or negatively orthant dependent, ND in short) if

$$
\begin{aligned}
& P\left(X_{1}>x_{1}, X_{2}>x_{2}, \ldots, X_{n}>x_{n}\right) \leq \prod_{i=1}^{n} P\left(X_{i}>x_{i}\right), \\
& P\left(X_{1} \leq x_{1}, X_{2} \leq x_{2}, \ldots, X_{n} \leq x_{n}\right) \leq \prod_{i=1}^{n} P\left(X_{i} \leq x_{i}\right)
\end{aligned}
$$

hold for all $x_{1}, x_{2}, \ldots, x_{n} \in \mathbb{R}$. An infinite sequence $\left\{X_{n}, n \geq\right.$ $1\}$ is said to be ND if every finite subcollection is ND.

Obviously, independent random variables are ND. JoagDev and Proschan [15] pointed out that negatively associated (NA, in short) random variables are ND. They also presented an example in which $X=\left(X_{1}, X_{2}, X_{3}, X_{4}\right)$ possesses ND but does not possess NA. The another example which is ND but is not NA was provided by $\mathrm{Wu}[16]$ as follows.

Example 1. Let $X_{i}$ be a binary random variable such that $P\left(X_{i}=1\right)=P\left(X_{i}=0\right)=0.5$ for $i=1,2,3$. Let $\left(X_{1}, X_{2}, X_{3}\right)$ take the values $(0,0,1),(0,1,0),(1,0,0)$, and $(1,1,1)$, each with probability $1 / 4$. It can be verified that all the ND conditions hold. However,

$$
\begin{aligned}
& P\left(X_{1}+X 3 \leq 1, X_{2} \leq 0\right) \\
& \quad=\frac{4}{8} \not P\left(X_{1}+X 3 \leq 1\right) P\left(X_{2} \leq 0\right)=\frac{3}{8} .
\end{aligned}
$$

Hence, $X_{1}, X_{2}$, and $X_{3}$ are not NA.

So we can see that ND is weaker than NA. A number of well-known multivariate distributions have the ND properties, such as multinomial, convolution of unlike multinomials, multivariate hypergeometric, dirichlet, dirichlet compound multinomial, and multinomials having certain covariance matrices. Because of the wide applications of ND random variables, the limiting behaviors of ND random variables have received more and more attention recently. A number of useful results for ND random variables have been established by many authors. We refer to Volodin [17] for the Kolmogorov exponential inequality, Asadian et al. [18] for Rosenthal's type inequality, Kim [19] for Hájek-Rényi type inequality, Amini et al. [20, 21], Ko and Kim [22], Klesov et al. [23], and Wang et al. [24] for almost sure convergence, Amini and Bozorgnia [25], Kuczmaszewska [26], Taylor et al. [27], Zarei and Jabbari [28], Wu [16, 29], Sung [30], and Wang et al. [31] for complete convergence, Wang et al. [32] for exponential inequalities and inverse moment, Shen [33] for strong limit theorems for arrays of rowwise ND random variables, Shen [34] for strong convergence rate for weighted sums of arrays of rowwise ND random variables, and so on. When these are compared with the corresponding results of independent random variable sequences, there still remains much to be desired.
This work is organized as follows: some preliminary lemmas are presented in Section 2, and the main results and their proofs are provided in Section 3.

Throughout the paper, $C$ denotes a positive constant not depending on $n$, which may be different in various places. $a_{n}=O\left(b_{n}\right)$ represents $a_{n} \leq C b_{n}$ for all $n \geq 1$. Let $[x]$ denote the integer part of $x$, and let $I(A)$ be the indicator function of the set $A$. Denote $x^{+}=x I(x \geq 0)$ and $x^{-}=-x I(x<0)$.

\section{Preliminaries}

In this section, we will present some important lemmas which will be used to prove the main results of the paper.

Lemma 2 (cf. Bozorgnia et al. [35]). Let the random variables $X_{1}, X_{2}, \ldots, X_{n}$ be ND, and let $f_{1}, f_{2}, \ldots, f_{n}$ be all nondecreasing (or all nonincreasing) functions. Then random variables $f_{1}\left(X_{1}\right), f_{2}\left(X_{2}\right), \ldots, f_{n}\left(X_{n}\right)$ are ND.

Lemma 3 (cf. Asadian et al. [18]). Let $p \geq 2$, and let $\left\{X_{n}, n \geq\right.$ $1\}$ be a sequence of $N D$ random variables with $E X_{n}=0$ and $E\left|X_{n}\right|^{p}<\infty$ for every $n \geq 1$. Then there exists a positive constant $C$ depending only on $p$ such that for every $n \geq 1$,

$$
E\left|\sum_{i=1}^{n} X_{i}\right|^{p} \leq C\left\{\sum_{i=1}^{n} E\left|X_{i}\right|^{p}+\left(\sum_{i=1}^{n} E X_{i}^{2}\right)^{p / 2}\right\} .
$$

The following concept of stochastic domination will be used in this work.

Definition 4. A sequence $\left\{X_{n}, n \geq 1\right\}$ of random variables is said to be stochastically dominated by a random variable $X$ if there exists a positive constant $C$ such that

$$
P\left(\left|X_{n}\right|>x\right) \leq C P(|X|>x)
$$

for all $x \geq 0$ and $n \geq 1$.

By the definition of stochastic domination and integration by parts, we can get the following property for stochastic domination. For the details of the proof, one can refer to $\mathrm{Wu}$ $[36,37]$ or Shen and $\mathrm{Wu}[38]$.

Lemma 5. Let $\left\{X_{n}, n \geq 1\right\}$ be a sequence of random variables which is stochastically dominated by a random variable $X$. For any $\alpha>0$ and $b>0$, the following two statements hold:

$$
\begin{gathered}
E\left|X_{n}\right|^{\alpha} I\left(\left|X_{n}\right| \leq b\right) \leq C_{1}\left[E|X|^{\alpha} I(|X| \leq b)+b^{\alpha} P(|X|>b)\right], \\
E\left|X_{n}\right|^{\alpha} I\left(\left|X_{n}\right|>b\right) \leq C_{2} E|X|^{\alpha} I(|X|>b),
\end{gathered}
$$

where $C_{1}$ and $C_{2}$ are positive constants.

\section{Main Results and Their Proofs}

Unless otherwise specified, we assume throughout the paper that $g_{n}(x)$ is defined by (2). For any function $g(x)$, we use $c(g)$ to denote all continuity points of the function $g$ on $A$. 
The norm $\|x\|$ is the Eucledean norm. For any fixed design point $x \in A$, the following assumptions on weight function $W_{n i}(x)$ will be used:

$$
\begin{aligned}
& \left(\mathrm{A}_{1}\right) \sum_{i=1}^{n} W_{n i}(x) \rightarrow 1 \text { as } n \rightarrow \infty ; \\
& \left(\mathrm{A}_{2}\right) \sum_{i=1}^{n}\left|W_{n i}(x)\right| \leq C<\infty \text { for all } n ; \\
& \left(\mathrm{A}_{3}\right) \sum_{i=1}^{n}\left|W_{n i}(x)\right| \cdot\left|g\left(x_{n i}\right)-g(x)\right| I\left(\left\|x_{n i}-x\right\|>a\right) \rightarrow 0 \text { as } \\
& \\
& n \rightarrow \infty \text { for all } a>0 .
\end{aligned}
$$

Based on the assumptions above, we can get the following strong consistency of the fixed design regression estimator $g_{n}(x)$.

Theorem 6. Let $\left\{\varepsilon_{n}, n \geq 1\right\}$ be a sequence of mean zero ND random variables, which is stochastically dominated by a random variable $X$. Assume that conditions $\left(A_{1}\right)-\left(A_{3}\right)$ hold true. If $E|X|^{p}<\infty$ for some $p>1$, and if there exist some $s \in(1 / p, 1)$ such that

$$
\max _{1 \leq i \leq n}\left|W_{n i}(x)\right|=O\left(n^{-s}\right),
$$

then for any $x \in c(g)$,

$$
g_{n}(x) \longrightarrow g(x) \text { a.s., } \quad \text { as } n \longrightarrow \infty \text {. }
$$

Proof. For $x \in c(g)$ and $a>0$, we have by (1) and (2) that

$$
\begin{aligned}
& \left|E g_{n}(x)-g(x)\right| \\
& \leq \sum_{i=1}^{n}\left|W_{n i}(x)\right| \cdot\left|g\left(x_{n i}\right)-g(x)\right| I\left(\left\|x_{n i}-x\right\| \leq a\right) \\
& \quad+\sum_{i=1}^{n}\left|W_{n i}(x)\right| \cdot\left|g\left(x_{n i}\right)-g(x)\right| I\left(\left\|x_{n i}-x\right\|>a\right) \\
& \quad+|g(x)| \cdot\left|\sum_{i=1}^{n} W_{n i}(x)-1\right| .
\end{aligned}
$$

Since $x \in c(g)$, hence for any $\varepsilon>0$, there exist a $\delta>0$ and $\left\|x^{*}-x\right\|<\delta$ such that $\left|g\left(x^{*}\right)-g(x)\right|<\varepsilon$. If we take $a \in(0, \delta)$ in (10), we can get that

$$
\begin{aligned}
& \left|E g_{n}(x)-g(x)\right| \\
& \leq \varepsilon \sum_{i=1}^{n}\left|W_{n i}(x)\right|+|g(x)| \cdot\left|\sum_{i=1}^{n} W_{n i}(x)-1\right| \\
& \quad+\sum_{i=1}^{n}\left|W_{n i}(x)\right| \cdot\left|g\left(x_{n i}\right)-g(x)\right| I\left(\left\|x_{n i}-x\right\|>a\right) .
\end{aligned}
$$

Therefore, we have by conditions $\left(\mathrm{A}_{1}\right)-\left(\mathrm{A}_{3}\right)$ that

$$
\lim _{n \rightarrow \infty} E g_{n}(x)=g(x), \quad x \in c(g) .
$$

For a fixed design point $x \in c(g)$, without loss of generality, we assume that $W_{n i}(x) \geq 0$ (otherwise, we use $W_{n i}^{+}(x)$ and $W_{n i}^{-}(x)$ instead of $W_{n i}(x)$, resp., and note that $W_{n i}(x)=$ $\left.W_{n i}^{+}(x)-W_{n i}^{-}(x)\right)$.
By (12), we can see that in order to prove (9), we only need to show that

$$
\begin{aligned}
g_{n}(x)-E g_{n}(x) & =\sum_{i=1}^{n} W_{n i}(x) \varepsilon_{n i} \\
& \doteq \sum_{i=1}^{n} R_{n i} \longrightarrow 0 \text { a.s., as } n \longrightarrow \infty,
\end{aligned}
$$

where $R_{n i}=W_{n i}(x) \varepsilon_{n i}$.

For fixed $\varepsilon>0$, choose $1 / p<\delta<s$ and some positive integer $N$ (to be specified later). Denote, for $i=1,2, \ldots, n$,

$$
\begin{aligned}
X_{n i}(1)= & -n^{-\delta} I\left(R_{n i}<-n^{-\delta}\right)+R_{n i} I\left(\left|R_{n i}\right| \leq n^{-\delta}\right) \\
& +n^{-\delta} I\left(R_{n i}>n^{-\delta}\right) \\
X_{n i}(2)= & \left(R_{n i}-n^{-\delta}\right) I\left(n^{-\delta}<R_{n i}<\frac{\varepsilon}{N}\right) \\
X_{n i}(3)= & \left(R_{n i}+n^{-\delta}\right) I\left(-n^{-\delta}>R_{n i}>-\frac{\varepsilon}{N}\right) \\
X_{n i}(4)= & \left(R_{n i}-n^{-\delta}\right) I\left(R_{n i} \geq \frac{\varepsilon}{N}\right) \\
& +\left(R_{n i}+n^{-\delta}\right) I\left(R_{n i} \leq-\frac{\varepsilon}{N}\right) .
\end{aligned}
$$

It is easy to check that $X_{n i}(1)+X_{n i}(2)+X_{n i}(3)+X_{n i}(4)=R_{n i}$. Hence, to prove (13), it suffices to show that, for any $x \in c(g)$,

$$
\sum_{i=1}^{n} X_{n i}(j) \longrightarrow 0 \text { a.s., } \quad \text { as } n \longrightarrow \infty, j=1,2,3,4 \text {. }
$$

By $E \varepsilon_{n}=0$ and $E|X|^{p}<\infty$, we can see that

$$
\begin{aligned}
& \left|\sum_{i=1}^{n} E X_{n i}(1)\right| \\
& \quad \leq \sum_{i=1}^{n}\left[n^{-\delta} P\left(\left|R_{n i}\right|>n^{-\delta}\right)+E\left|R_{n i}\right| I\left(\left|R_{n i}\right|>n^{-\delta}\right)\right] \\
& \quad \leq \sum_{i=1}^{n} n^{-\delta} \frac{E\left|R_{n i}\right|^{p}}{n^{-p \delta}} \leq C n^{\delta(p-1)} \sum_{i=1}^{n}\left|W_{n i}(x)\right|^{p} E|X|^{p} \\
& \quad \leq C n^{\delta(p-1)}\left(\max _{1 \leq i \leq n}\left|W_{n i}(x)\right|\right)^{p-1} \sum_{i=1}^{n}\left|W_{n i}(x)\right| \\
& \quad \leq C n^{-(p-1)(s-\delta)} \longrightarrow 0, \quad \text { as } n \longrightarrow \infty .
\end{aligned}
$$

Hence, to prove $\sum_{i=1}^{n} X_{n i}(1) \rightarrow 0$ a.s., it suffices to show that

$$
\sum_{n=1}^{\infty} P\left(\left|\sum_{i=1}^{n}\left(X_{n i}(1)-E X_{n i}(1)\right)\right|>\varepsilon\right)<\infty .
$$


It is easily seen that, for fixed $n \geq 1$ and $x \in c(g),\left\{X_{n i}(1), 1 \leq\right.$ $i \leq n\}$ are still ND random variables by Lemma 2. Applying Lemma 3, we have for $q \geq 2$ that

$$
\begin{aligned}
& \sum_{n=1}^{\infty} P\left(\left|\sum_{i=1}^{n}\left(X_{n i}(1)-E X_{n i}(1)\right)\right|>\varepsilon\right) \\
& \leq C \sum_{n=1}^{\infty} \sum_{i=1}^{n} E\left|X_{n i}(1)\right|^{q}+C \sum_{n=1}^{\infty}\left(\sum_{i=1}^{n} E\left|X_{n i}(1)\right|^{2}\right)^{q / 2} \\
& \quad \doteq C I_{11}+C I_{12} .
\end{aligned}
$$

Taking $q>\max \{p,(s+1+p(\delta-s)) / \delta, 2 /((s-\delta)(p-1)+$ $\delta), 2 / s\}$, we can get that

$$
\begin{gathered}
(q-p) \delta+s(p-1)>1, \quad \frac{q s}{2}>1 \\
\frac{[(2-p) \delta+s(p-1)] q}{2}=\frac{[(s-\delta)(p-1)+\delta] q}{2}>1 .
\end{gathered}
$$

For $I_{11}$, we have by $C_{r}$ 's inequality, Lemma 5 , and condition $\left(\mathrm{A}_{2}\right)$ that

$$
\begin{aligned}
& I_{11} \leq C \sum_{n=1}^{\infty} \sum_{i=1}^{n}\left[n^{-q \delta} P\left(\left|R_{n i}\right|>n^{-\delta}\right)\right.\left.+E\left|R_{n i}\right|^{q} I\left(\left|R_{n i}\right| \leq n^{-\delta}\right)\right] \\
& \leq C \sum_{n=1}^{\infty} \sum_{i=1}^{n}\left[n^{-q \delta} P\left(\left|W_{n i}(x) X\right|>n^{-\delta}\right)\right. \\
&\left.\quad+E\left|W_{n i}(x) X\right|^{q} I\left(\left|W_{n i}(x) X\right| \leq n^{-\delta}\right)\right] \\
& \leq C \sum_{n=1}^{\infty} \sum_{i=1}^{n} n^{-q \delta} \frac{E\left|W_{n i}(x) X\right|^{p}}{n^{-p \delta}} \\
& \leq C \sum_{n=1}^{\infty} n^{-(q-p) \delta}\left(\max _{1 \leq i \leq n}\left|W_{n i}(x)\right|\right)^{p-1} \times \sum_{i=1}^{n}\left|W_{n i}(x)\right| \\
& \leq C \sum_{n=1}^{\infty} n^{-[(q-p) \delta+s(p-1)]}<\infty .
\end{aligned}
$$

For $I_{12}$, if $1<p<2$, we have by the proof of (20) that

$$
\begin{aligned}
& I_{12} \leq C \sum_{n=1}^{\infty}\left\{\sum_{i=1}^{n}[\right. n^{-2 \delta} P\left(\left|R_{n i}\right|>n^{-\delta}\right) \\
&\left.\left.+E\left|R_{n i}\right|^{2} I\left(\left|R_{n i}\right| \leq n^{-\delta}\right)\right]\right\}^{q / 2} \\
& \leq C \sum_{n=1}^{\infty} n^{-[(2-p) \delta+s(p-1)] q / 2}<\infty
\end{aligned}
$$

if $p \geq 2$, we have by $E X^{2}<\infty$ that

$$
\begin{gathered}
I_{12} \leq C \sum_{n=1}^{\infty}\left\{\sum _ { i = 1 } ^ { n } \left[n^{-2 \delta} P\left(\left|R_{n i}\right|>n^{-\delta}\right)\right.\right. \\
\left.\left.+E\left|R_{n i}\right|^{2} I\left(\left|R_{n i}\right| \leq n^{-\delta}\right)\right]\right\}^{q / 2} \\
\leq C \sum_{n=1}^{\infty}\left\{\sum_{i=1}^{n}\left[n^{-2 \delta} P\left(\left|W_{n i}(x) X\right|>n^{-\delta}\right)+W_{n i}^{2}(x)\right]\right\}^{q / 2} \\
\leq C \sum_{n=1}^{\infty} n^{-[(2-p) \delta+s(p-1)] q / 2}+C \sum_{n=1}^{\infty} n^{-q s / 2}<\infty .
\end{gathered}
$$

By (18)-(22), we can get (17), which together with (16) imply that $\sum_{i=1}^{n} X_{n i}(1) \rightarrow 0$ a.s.

Next, we will prove that $\sum_{i=1}^{n} X_{n i}(2) \rightarrow 0$ a.s. Since $0 \leq$ $X_{n i}(2)<\varepsilon / N,\left|\sum_{i=1}^{n} X_{n i}(2)\right|=\sum_{i=1}^{n} X_{n i}(2)>\varepsilon$ implies that there are at least $N i$ s such that $X_{n i}(2) \neq 0$. Hence,

$$
P\left(\left|\sum_{i=1}^{n} X_{n i}(2)\right|>\varepsilon\right)
$$

$\leq P\left(\right.$ there are at least $N i$ 's such that $\left.X_{n i}(2) \neq 0\right)$

$$
\begin{aligned}
& \leq \sum_{1 \leq i_{1}<i_{2}<\cdots<i_{N} \leq n} P\left(X_{n i_{1}}(2) \neq 0, X_{n i_{2}}(2) \neq 0,\right. \\
& \leq \sum_{1 \leq i_{1}<i_{2}<\cdots<i_{N} \leq n} P\left(R_{n i_{1}}>n^{-\delta}, R_{n i_{N}}>n^{-\delta}, \ldots, R_{n i_{N}}>n^{-\delta}\right) \\
& \leq \sum_{1 \leq i_{1}<i_{2}<\cdots<i_{N} \leq n} P\left(R_{n i_{1}}>n^{-\delta}\right) P\left(R_{n i_{2}}>n^{-\delta}\right) \\
& \leq\left[\sum_{i=1}^{n} P\left(R_{n i}>n^{-\delta}\right)\right]^{N} \leq\left[\sum_{i=1}^{n} P\left(\left|R_{n i}\right|>n^{-\delta}\right)\right]^{N} \\
& \left.\leq C\left[\sum_{i=1}^{n} \frac{E\left|W_{n i}(x) X\right|^{p}}{n^{-p \delta}}\right]^{N}>n^{-\delta}\right) \\
& \leq C\left[n^{p \delta}\left(\max _{1 \leq i \leq n}\left|W_{n i}(x)\right|\right)^{p-1} \sum_{i=1}^{n}\left|W_{n i}(x)\right|\right]^{N} \\
& \leq C n^{-[(s-\delta) p+s] N},
\end{aligned}
$$

which is summable if we choose large integer $N$ such that [ $(s-$ $\delta) p+s] N>1$. Hence, $\sum_{i=1}^{n} X_{n i}(2) \rightarrow 0$ a.s. by Borel-Cantelli lemma.

Since $-\varepsilon / N<X_{n i}(3) \leq 0,\left|\sum_{i=1}^{n} X_{n i}(3)\right|=$ $-\sum_{i=1}^{n} X_{n i}(3)>\varepsilon$ implies that there are at least $N$ i's such that $X_{n i}(3) \neq 0$. Similarly, we have $\sum_{i=1}^{n} X_{n i}(3) \rightarrow 0$ a.s. as $n \rightarrow \infty$. 
Finally, we will prove that $\sum_{i=1}^{n} X_{n i}(4) \rightarrow 0$ a.s. as $n \rightarrow$ $\infty$. It is easily seen that

$$
\begin{aligned}
& \left|\sum_{i=1}^{n} X_{n i}(4)\right| \\
& \quad \leq \sum_{i=1}^{n}\left|R_{n i}\right| I\left(\left|R_{n i}\right| \geq \frac{\varepsilon}{N}\right)+n^{-\delta} \sum_{i=1}^{n} I\left(\left|R_{n i}\right| \geq \frac{\varepsilon}{N}\right) \\
& \quad \leq C n^{-s} \sum_{i=1}^{n}\left|\varepsilon_{n i}\right| I\left(\left|\varepsilon_{n i}\right| \geq C n^{s}\right)+n^{-\delta} \sum_{i=1}^{n} I\left(\left|\varepsilon_{n i}\right| \geq C n^{s}\right) \\
& \quad \leq C n^{-s} \sum_{i=1}^{n}\left|\varepsilon_{n i}\right| I\left(\left|\varepsilon_{n i}\right| \geq C i^{s}\right)+n^{-\delta} \sum_{i=1}^{n} I\left(\left|\varepsilon_{n i}\right| \geq C i^{s}\right) \\
& \quad \leq H_{n 1}+H_{n 2} .
\end{aligned}
$$

To prove that $\sum_{i=1}^{n} X_{n i}(4) \rightarrow 0$ a.s. as $n \rightarrow \infty$, it suffices to show that $H_{n 1} \rightarrow 0$ a.s. and $H_{n 2} \rightarrow 0$ a.s. as $n \rightarrow \infty$. Firstly, we will show that

$$
\begin{gathered}
\sum_{i=1}^{\infty} i^{-s}\left|\varepsilon_{n i}\right| I\left(\left|\varepsilon_{n i}\right| \geq C i^{s}\right)<\infty \text { a.s. } \\
\sum_{i=1}^{\infty} i^{-\delta} I\left(\left|\varepsilon_{n i}\right| \geq C i^{s}\right)<\infty \text { a.s. }
\end{gathered}
$$

Denote that $T_{m}=\sum_{i=1}^{m} i^{-s}\left|\varepsilon_{n i}\right| I\left(\left|\varepsilon_{n i}\right| \geq C i^{s}\right)$. For $m \geq k \geq 1$, it follows by Lemma 5 and $E|X|^{p}<\infty$ that

$$
\begin{aligned}
E\left|T_{m}-T_{k}\right| & =\sum_{i=k+1}^{m} i^{-s} E\left|\varepsilon_{n i}\right| I\left(\left|\varepsilon_{n i}\right| \geq C i^{s}\right) \\
& \leq C \sum_{i=k+1}^{m} i^{-s} E|X| I\left(|X| \geq C i^{s}\right) \\
& \leq C \sum_{i=k+1}^{m} i^{-s p} \\
& \leq C k^{-s p+1} \longrightarrow 0 \text { as } k \longrightarrow \infty
\end{aligned}
$$

which implies that $\left\{T_{m}, m \geq 1\right\}$ is a Cauchy sequence in $L_{1}$, and hence there exists a random variable $T$ such that $E|T|<$ $\infty$ and $E\left|T_{m}-T\right| \rightarrow 0$. It follows by Lemma 5 and $E|X|^{p}<\infty$ again that

$$
\begin{aligned}
& P\left(\left|T_{2^{k}}-T\right|>\varepsilon\right) \\
& \leq C E\left|T_{2^{k}}-T\right| \leq C \limsup _{m \rightarrow \infty} E\left|T_{2^{k}}-T_{m}\right| \\
& \leq C \sum_{i=2^{k}+1}^{\infty} i^{-s} E\left|\varepsilon_{n i}\right| I\left(\left|\varepsilon_{n i}\right| \geq C i^{s}\right) \\
& \leq C \sum_{i=2^{k}+1}^{\infty} i^{-s} E|X| I\left(|X| \geq C i^{s}\right) \\
& \leq C \sum_{i=2^{k}+1}^{\infty} i^{-s p} \leq C 2^{-k(s p-1)},
\end{aligned}
$$

$$
\begin{aligned}
& P\left(\max _{2^{k-1}<m \leq 2^{k}}\left|T_{m}-T_{2^{k-1}}\right|>\varepsilon\right) \\
& \leq C E\left(\max _{2^{k-1}<m \leq 2^{k}}\left|T_{m}-T_{2^{k-1}}\right|\right) \\
& \leq C \sum_{i=2^{k-1}+1}^{2^{k}} i^{-s} E\left|\varepsilon_{n i}\right| I\left(\left|\varepsilon_{n i}\right| \geq C i^{s}\right) \\
& \leq C \sum_{i=2^{k-1}+1}^{2^{k}} i^{-s p} \leq C 2^{-k(s p-1)},
\end{aligned}
$$

which together imply that $T_{m} \rightarrow T$ a.s., and thus (25) holds. Similarly, we can get (26). Therefore, $H_{n 1} \rightarrow 0$ a.s. and $H_{n 2} \rightarrow 0$ a.s. follow by (25)-(26) and the Kronecker's lemma immediately. This completes the proof of the theorem.

As an application of Theorem 6, we give the strong consistency for the nearest neighbor estimator of $g(x)$. Without loss of generality, putting $A=[0,1]$, taking $x_{n i}=i / n$, $i=1,2, \ldots, n$, and $n \geq 1$. For any $x \in A$, we rewrite $\left|x_{n 1}-x\right|,\left|x_{n 2}-x\right|, \ldots,\left|x_{n n}-x\right|$ as follows:

$$
\left|x_{R_{1}(x)}^{(n)}-x\right| \leq\left|x_{R_{2}(x)}^{(n)}-x\right| \leq \cdots \leq\left|x_{R_{n}(x)}^{(n)}-x\right| .
$$

If $\left|x_{n i}-x\right|=\left|x_{n j}-x\right|$, then $\left|x_{n i}-x\right|$ is permuted before $\left|x_{n j}-x\right|$ when $x_{n i}<x_{n j}$.

Let $1 \leq k_{n} \leq n$, the nearest neighbor weight function estimator of $g(x)$ in model (1) is defined as follows:

$$
\widetilde{g}_{n}(x)=\sum_{i=1}^{n} \widetilde{W}_{n i}(x) Y_{n i}
$$

where

$$
\widetilde{W}_{n i}(x)= \begin{cases}\frac{1}{k_{n}}, & \text { if }\left|x_{n i}-x\right| \leq\left|x_{R_{k_{n}}(x)}^{(n)}-x\right| \\ 0, & \text { otherwise. }\end{cases}
$$

Based on the notations above, we can get the following result by using Theorem 6 .

Corollary 7. Let $\left\{\varepsilon_{n}, n \geq 1\right\}$ be a sequence of mean zero ND random variables, which is stochastically dominated by a random variable $X$. Assume that $g$ is continuous on the compact set $A$. If $E|X|^{p}<\infty$ for some $p>1$ and if there exists some $s \in(1 / p, 1)$ such that $k_{n}=\left[n^{s}\right]$, then for any $x \in c(g)$,

$$
\tilde{g}_{n}(x) \longrightarrow g(x) \text { a.s., } \quad \text { as } n \longrightarrow \infty \text {. }
$$

Proof. It suffices to show that the conditions of Theorem 6 are satisfied. Since $g$ is continuous on the compact set $A$, hence $g$ is uniformly continuous on the compact set $A$, which implies that $\left\{\left|g\left(x_{n i}\right)-g(x)\right|: 1 \leq i \leq n, n \geq 1\right\}$ is bounded on the set A. 
For any $x \in[0,1]$, if follows from the definition of $R_{i}(x)$ and $\widetilde{W}_{n i}(x)$ that

$$
\begin{aligned}
& \sum_{i=1}^{n} \widetilde{W}_{n i}(x)=\sum_{i=1}^{n} \widetilde{W}_{n R_{i}(x)}(x)=\sum_{i=1}^{k_{n}} \frac{1}{k_{n}}=1, \\
& \max _{1 \leq i \leq n} \widetilde{W}_{n i}(x)=\frac{1}{k_{n}}, \quad \widetilde{W}_{n i}(x) \geq 0, \\
& \sum_{i=1}^{n}\left|\widetilde{W}_{n i}(x)\right| \cdot\left|g\left(x_{n i}\right)-g(x)\right| I\left(\left|x_{n i}-x\right|>a\right) \\
& \quad \leq C \sum_{i=1}^{n} \frac{\left(x_{n i}-x\right)^{2}\left|\widetilde{W}_{n i}(x)\right|}{a^{2}} \\
& \quad=C \sum_{i=1}^{k_{n}} \frac{\left(x_{R_{i}(x)}^{(n)}-x\right)^{2}}{k_{n} a^{2}} \leq C \sum_{i=1}^{\frac{k_{n}}{(i / n)^{2}}} \frac{k_{n} a^{2}}{} \\
& \quad \leq C\left(\frac{k_{n}}{n a}\right)^{2}, \quad \forall a>0 .
\end{aligned}
$$

Hence, conditions $\left(A_{1}\right)-\left(A_{3}\right)$ and (8) are satisfied. By Theorem 6, we can get (32) immediately. This completes the proof of the corollary.

\section{Acknowledgments}

The authors are most grateful to the Editor Michiel Bertsch and anonymous referee for careful reading of the paper and valuable suggestions which helped in improving an earlier version of this paper. This work was supported by the National Natural Science Foundation of China (11201001, 11171001), the Natural Science Foundation of Anhui Province (1208085QA03, 1308085QA03), Applied Teaching Model Curriculum of Anhui University (XJYYXKC04), Doctoral Research Start-up Funds Projects of Anhui University, Students Innovative Training Project of Anhui University (201310357004), and the Students Science Research Training Program of Anhui University (KYXL2012007, kyxl2013003).

\section{References}

[1] A. A. Georgiev, "Local properties of function fitting estimates with applications to system identification," in Mathematical Statistics and Applications, W. Grossmann et al., Ed., Volume 2 of Proceedings of the 4th Pannonian Symposium on Mathematical Statistics, Bad Tatzmannsdorf, Austria, 4-10 September 1983, pp. 141-151, Reidel, Dordrecht, The Netherlands, 1985.

[2] A. A. Georgiev and W. Greblicki, "Nonparametric function recovering from noisy observations," Journal of Statistical Planning and Inference, vol. 13, no. 1, pp. 1-14, 1986.

[3] A. A. Georgiev, "Consistent nonparametric multiple regression: the fixed design case," Journal of Multivariate Analysis, vol. 25, no. 1, pp. 100-110, 1988.

[4] H. G. Müller, "Weak and universal consistency of moving weighted averages," Periodica Mathematica Hungarica, vol. 18, no. 3, pp. 241-250, 1987.
[5] Y. Fan, "Consistent nonparametric multiple regression for dependent heterogeneous processes: the fixed design case," Journal of Multivariate Analysis, vol. 33, no. 1, pp. 72-88, 1990.

[6] G. G. Roussas, "Consistent regression estimation with fixed design points under dependence conditions," Statistics \& Probability Letters, vol. 8, no. 1, pp. 41-50, 1989.

[7] G. G. Roussas, L. T. Tran, and D. A. Ioannides, "Fixed design regression for time series: asymptotic normality," Journal of Multivariate Analysis, vol. 40, no. 2, pp. 262-291, 1992.

[8] L. Tran, G. Roussas, S. Yakowitz, and B. Truong Van, "Fixeddesign regression for linear time series," The Annals of Statistics, vol. 24, no. 3, pp. 975-991, 1996.

[9] S. H. Hu, C. H. Zhu, Y. B. Chen, and L. C. Wang, "Fixed-design regression for linear time series," Acta Mathematica Scientia B, vol. 22, no. 1, pp. 9-18, 2002.

[10] S. H. Hu, G. M. Pan, and Q. B. Gao, "Estimation problems for a regression model with linear process errors," Applied Mathematics-A Journal of Chinese Universities, vol. 18, no. 1, pp. 81-90, 2003.

[11] H.-Y. Liang and B.-Y. Jing, "Asymptotic properties for estimates of nonparametric regression models based on negatively associated sequences," Journal of Multivariate Analysis, vol. 95, no. 2, pp. 227-245, 2005.

[12] W. Z. Yang, X. J. Wang, X. H. Wang, and S. H. Hu, "The consistency for estimator of nonparametric regression model based on NOD errors," Journal of Inequalities and Applications, vol. 2012, article 140, 2012.

[13] A. T. Shen, "Bernstein-type inequality for widely dependent sequence and its application to nonparametric regression models," Abstract and Applied Analysis, vol. 2013, Article ID 862602, 9 pages, 2013.

[14] E. L. Lehmann, "Some concepts of dependence," The Annals of Mathematical Statistics, vol. 37, no. 5, pp. 1137-1153, 1966.

[15] K. Joag-Dev and F. Proschan, "Negative association of random variables, with applications," The Annals of Statistics, vol. 11, no. 1, pp. 286-295, 1983.

[16] Q. Y. Wu, "Complete convergence for weighted sums of sequences of negatively dependent random variables," Journal of Probability and Statistics, vol. 2011, Article ID 202015, 16 pages, 2011.

[17] A. Volodin, "On the Kolmogorov exponential inequality for negatively dependent random variables," Pakistan Journal of Statistics, vol. 18, no. 2, pp. 249-253, 2002.

[18] N. Asadian, V. Fakoor, and A. Bozorgnia, "Rosenthal's type inequalities for negatively orthant dependent random variables," Journal of the Iranian Statistical Society, vol. 5, no. 1-2, pp. 66-75, 2006.

[19] H. C. Kim, “The Hájeck-Rènyi inequality for weighted sums of negatively orthant dependent random variables," International Journal of Contemporary Mathematical Sciences, vol. 1, no. 5-8, pp. 297-303, 2006.

[20] M. Amini, H. A. Azarnoosh, and A. Bozorgnia, "The strong law of large numbers for negatively dependent generalized Gaussian random variables," Stochastic Analysis and Applications, vol. 22, no. 4, pp. 893-901, 2004.

[21] M. Amini, H. Zarei, and A. Bozorgnia, "Some strong limit theorems of weighted sums for negatively dependent generalized Gaussian random variables," Statistics \& Probability Letters, vol. 77, no. 11, pp. 1106-1110, 2007. 
[22] M. H. Ko and T. S. Kim, "Almost sure convergence for weighted sums of negatively orthant dependent random variables," Journal of the Korean Mathematical Society, vol. 42, no. 5, pp. 949957, 2005.

[23] O. Klesov, A. Rosalsky, and A. I. Volodin, "On the almost sure growth rate of sums of lower negatively dependent nonnegative random variables," Statistics \& Probability Letters, vol. 71, no. 2, pp. 193-202, 2005.

[24] X. J. Wang, S. H. Hu, A. T. Shen, and W. Z. Yang, "An exponential inequality for a NOD sequence and a strong law of large numbers," Applied Mathematics Letters, vol. 24, no. 2, pp. 219223, 2011.

[25] M. Amini and A. Bozorgnia, "Complete convergence for negatively dependent random variables," Journal of Applied Mathematics and Stochastic Analysis, vol. 16, no. 2, pp. 121-126, 2003.

[26] A. Kuczmaszewska, "On some conditions for complete convergence for arrays of rowwise negatively dependent random variables," Stochastic Analysis and Applications, vol. 24, no. 6, pp. 1083-1095, 2006.

[27] R. L. Taylor, R. F. Patterson, and A. Bozorgnia, "A strong law of large numbers for arrays of rowwise negatively dependent random variables," Stochastic Analysis and Applications, vol. 20, no. 3, pp. 643-656, 2002.

[28] H. Zarei and H. Jabbari, "Complete convergence of weighted sums under negative dependence," Statistical Papers, vol. 52, no. 2, pp. 413-418, 2011.

[29] Q. Y. Wu, "Complete convergence for negatively dependent sequences of random variables," Journal of Inequalities and Applications, vol. 2010, Article ID 507293, 10 pages, 2010.

[30] S. H. Sung, "Complete convergence for weighted sums of negatively dependent random variables," Statistical Papers, vol. 53, no. 1, pp. 73-82, 2012.

[31] X. J. Wang, S. H. Hu, and W. Z. Yang, "Complete convergence for arrays of rowwise negatively orthant dependent random variables," Revista de la Real Academia de Ciencias Exactas, Físicas y Naturales A, vol. 106, no. 2, pp. 235-245, 2012.

[32] X. J. Wang, S. H. Hu, W. Z. Yang, and N. X. Ling, "Exponential inequalities and inverse moment for NOD sequence," Statistics \& Probability Letters, vol. 80, no. 5-6, pp. 452-461, 2010.

[33] A. T. Shen, "Some strong limit theorems for arrays of rowwise negatively orthant-dependent random variables," Journal of Inequalities and Applications, vol. 2011, article 93, 2011.

[34] A. T. Shen, "On the strong convergence rate for weighted sums of arrays of rowwise negatively orthant dependent random variables," Revista de la Real Academia de Ciencias Exactas, Fisicas y Naturales A, vol. 107, no. 2, pp. 257-271, 2013.

[35] A. Bozorgnia, R. F. Patterson, and R. L. Taylor, "Limit theorems for dependent random variables," in Proceeding of the World Congress Nonlinear Analysts (WCNA '92), vol. 1, pp. 1639-1650, de Gruyter, Berlin, Germany, 1992.

[36] Q. Y. Wu, "A strong limit theorem for weighted sums of sequences of negatively dependent random variables," Journal of Inequalities and Applications, vol. 2010, Article ID 383805, 8 pages, 2010.

[37] Q. Y. Wu, "A complete convergence theorem for weighted sums of arrays of rowwise negatively dependent random variables," Journal of Inequalities and Applications, vol. 2012, article 50, 2012.

[38] A. T. Shen and R. C. Wu, "Strong and weak convergence for asymptotically almost negatively associated random variables,"
Discrete Dynamics in Nature and Society, vol. 2013, Article ID 235012, 7 pages, 2013. 


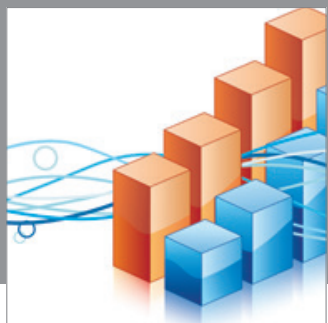

Advances in

Operations Research

mansans

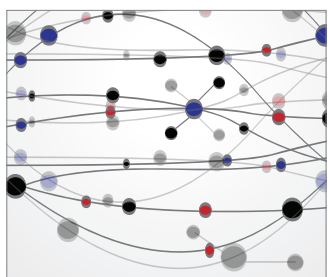

The Scientific World Journal
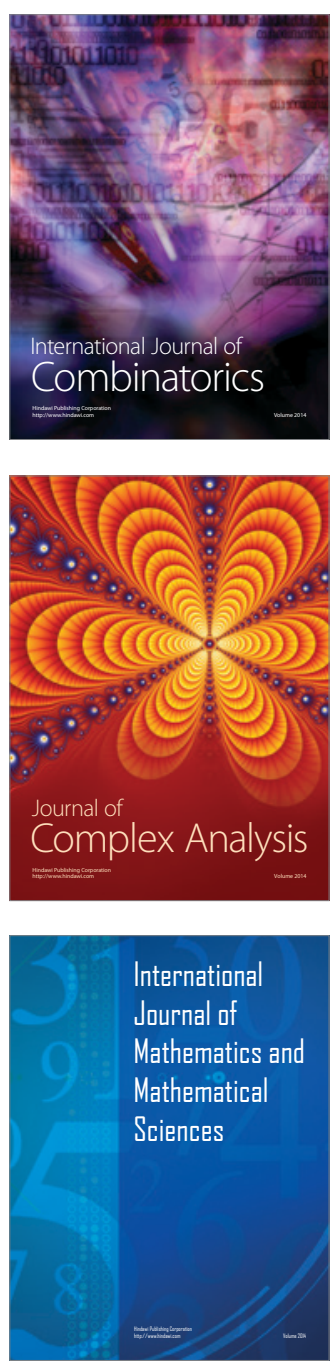
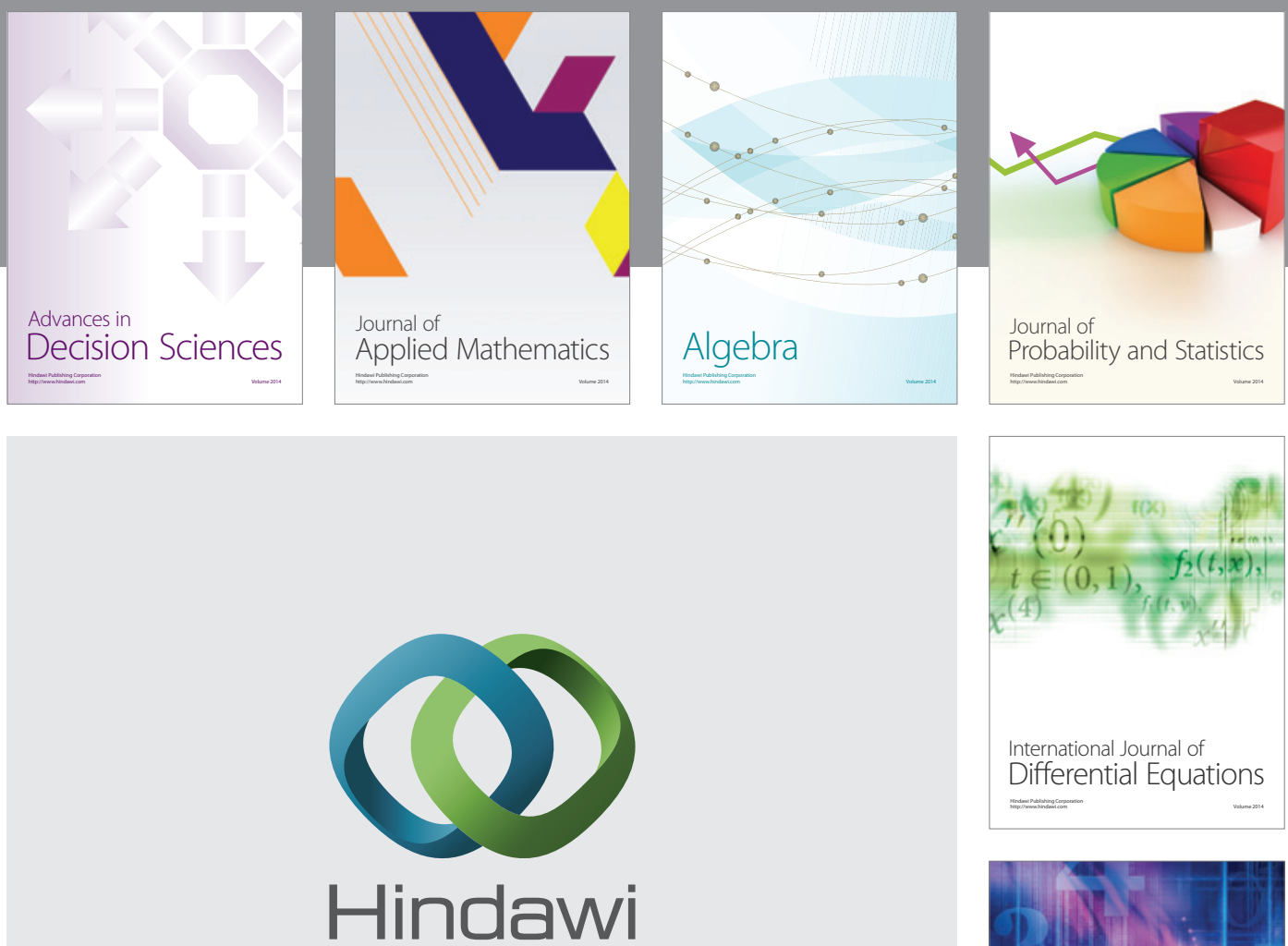

Submit your manuscripts at http://www.hindawi.com
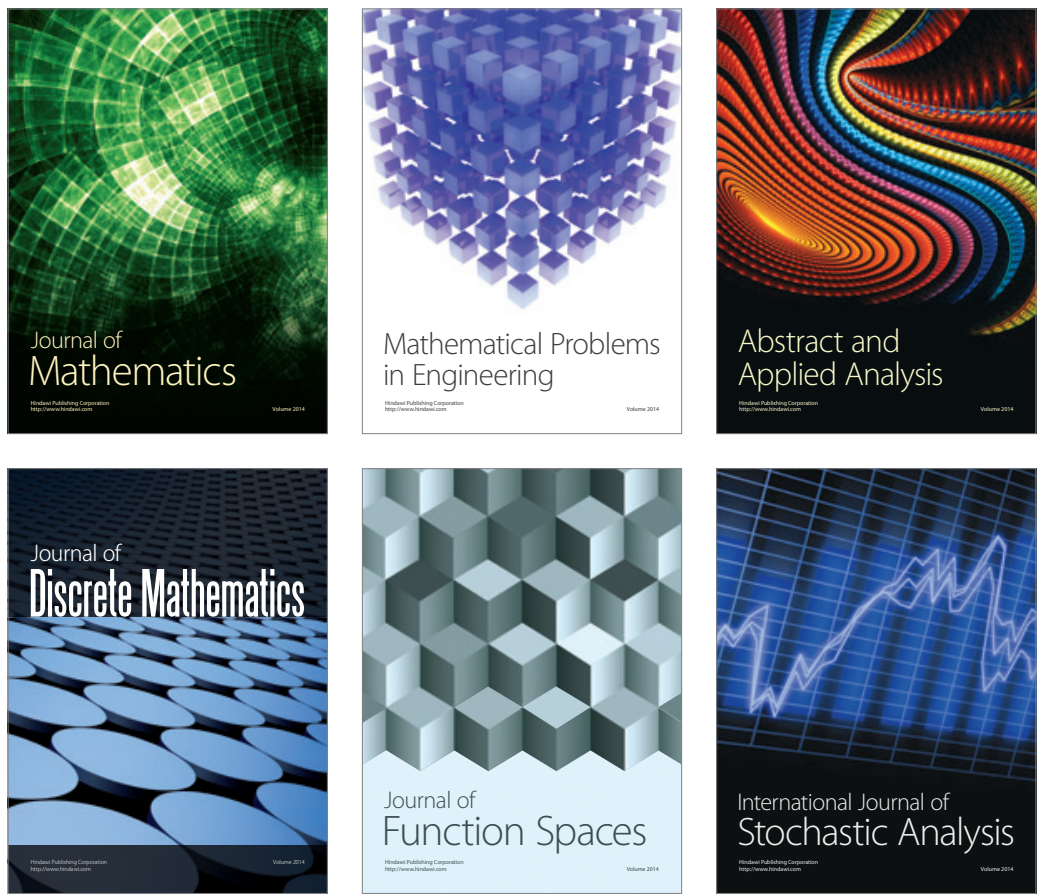

Journal of

Function Spaces

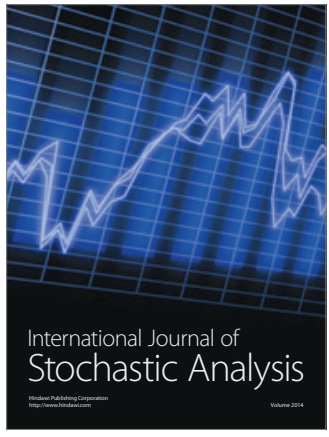

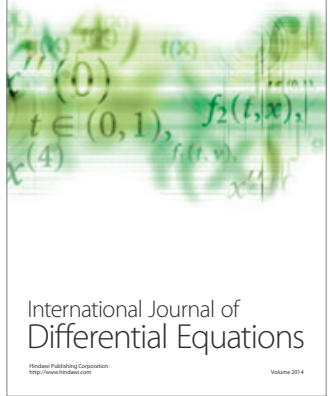
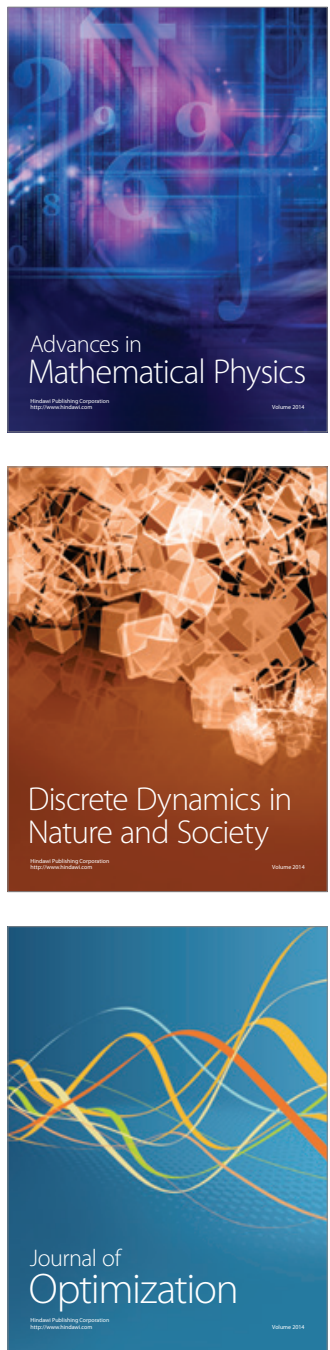